\title{
Q2 ANIMA: A data-sharing initiative for neuroimaging meta-analyses
}

\author{
Q3 Andrew T. Reid ${ }^{\mathrm{a}, *}$, Danilo Bzdok ${ }^{\mathrm{a}, \mathrm{b}, \mathrm{g}}$, Sarah Genon ${ }^{\mathrm{a}, \mathrm{b}}$, Robert Langner ${ }^{\mathrm{a}, \mathrm{b}}$, Veronika I. Müller ${ }^{\mathrm{a}, \mathrm{b}}$, \\ Claudia R. Eickhoff ${ }^{\mathrm{a}, \mathrm{c}}$, Felix Hoffstaedter ${ }^{\mathrm{a}, \mathrm{b}}$, Edna-Clarisse Cieslik ${ }^{\mathrm{a}, \mathrm{b}}$, Peter T. Fox ${ }^{\mathrm{d}}$, Angela R. Laird ${ }^{\mathrm{e}}$, \\ Katrin Amunts ${ }^{\mathrm{a}, \mathrm{f}}$, Simon B. Eickhoff ${ }^{\mathrm{a}, \mathrm{b}}$ \\ a Institute of Neuroscience and Medicine 1, Research Centre Jülich, Jülich, Germany \\ b Institute of Clinical Neuroscience and Medical Psychology, Heinrich Heine University, Düsseldorf, Germany \\ c Department of Psychiatry, Psychotherapy and Psychosomatics, University Hospital Aachen, Aachen, Germany \\ Q4 ${ }^{\mathrm{d}}$ University of Texas Health Sciences Center at San Antonio, San Antonio, TX, USA \\ e Florida International University, Miami, FL, USA \\ ${ }^{\mathrm{f}}$ C. E' O. Vogt Institute for Brain Research, Heinrich Heine University, Düsseldorf, Germany \\ ${ }^{g}$ INRIA, Neurospin, bat 145, CEA Saclay, 91191 Gif-sur-Yvette, France
}

\begin{abstract}
A B S T R A C T
Meta-analytic techniques allow cognitive neuroscientists to pool large amounts of data across many individual 17 task-based functional neuroimaging experiments. These methods have been aided by the introduction of online 18 databases such as Brainmap.org or Neurosynth.org, which collate peak activation coordinates obtained from 19 thousands of published studies. Findings from meta-analytic studies typically include brain regions which are 20 consistently activated across studies for specific contrasts, investigating cognitive or clinical hypotheses. These 21 regions can be subsequently used as the basis for seed-based connectivity analysis, or formally compared to neu- 22 roimaging data in order to help interpret new findings. To facilitate such approaches, we have developed a new 23 online repository of meta-analytic neuroimaging results, named the Archive of Neuroimaging Meta-analyses 24 (ANIMA). The ANIMA platform consists of an intuitive online interface for querying, downloading, and contrib- 25 uting data from published meta-analytic studies. Additionally, to aid the process of organizing, visualizing, and 26 working with these data, we present an open-source desktop application called Volume Viewer. Volume Viewer 27 allows users to easily arrange imaging data into composite stacks, and save these sessions as individual files, 28 which can also be uploaded to the ANIMA database. The application also allows users to perform basic functions, 29 such as computing conjunctions between images, or extracting regions-of-interest or peak coordinates for fur- 30 ther analysis. The introduction of this new resource will enhance the ability of researchers to both share their 31 findings and incorporate existing meta-analytic results into their own research.
\end{abstract}

(c) 2015 Published by Elsevier Inc.

\section{Introduction}

Functional neuroimaging, like many other scientific fields, is faced with the daunting task of managing an ever-increasing amount of data (Poldrack and Gorgolewski, 2014). Online databases such as Brainmap.org (Laird et al., 2011), Neurovault.org (Gorgolewski et al., 2015), and Neurosynth.org (Yarkoni et al., 2011) provide access to data from hundreds of published task-based fMRI studies, in standard coordinates (recently reviewed by Fox et al., 2014). In addition to extensive meta-analyses based upon manual search techniques, these platforms have facilitated a growing number of metaanalytic studies of the neural correlates of specific cognitive functions, using methods such as multilevel kernel density analysis (MKDA; Wager et al., 2007) and activation likelihood estimation (ALE; Eickhoff et al., 2009, 2012). Meta-analysis entails the pooling of data over tens

\footnotetext{
* Corresponding author. Fax: + 492461613483.

E-mail address: a.reid@fz-juelich.de (A.T. Reid).
}

to thousands of individual studies, and thus provides: (1.) greater 52 sensitivity and specificity to detect "true" effects; (2.) a means of deter- 53 mining core groups of brain regions subserving a specific task or charac- 54 terizing a specific disease; and (3.) a method for formal comparison of 55 different subfacets of a cognitive domain. This approach has been 56 used, for instance, to demonstrate the neural correlates of sustained at- 57 tention (Langner and Eickhoff, 2013), investigate face processing areas 58 in autistic subjects (Nickl-Jockschat et al., 2014), and identify key re- 59 gions subserving supervisory attentional control (Cieslik et al., 2015). 60

Results from meta-analyses can be subsequently used as robust prior 61 information in the design of task-based fMRI studies, and as regions-of- 62 interest (ROIs) for connectivity methods based on functional correla- 63 tions (e.g., Müller et al., 2014; Schilbach et al., 2014), or virtually any 64 other type of seed-based analysis. This includes topical meta-analytic 65 approaches such as ALE and MKDA, as well as methods which use 66 meta-analysis to infer functional connectivity, such as meta-analytic 67 connectivity modelling (MACM; Etkin and Wager, 2007; Kober et al., 68 2008; Robinson et al., 2010), in which functional coactivations are 69 
assessed across all tasks in the database (see also Xue et al., 2014). Additional meta-analytic approaches include the parcellation of the brain into functionally distinct subregions, such as coactivation-based parcellation (CBP; Chang et al., 2012; Eickhoff et al., 2011; Northoff et al., 2006). A typical ALE analysis, for example, will result in a distinct ROI or set of ROIs that are associated with a particular psychological or clinical feature (see Box 1). These results are then commonly used as seed regions for further analysis of these features (e.g., zu Eulenburg et al., 2012). Given their utility, results from meta-analytic studies are becoming increasingly popular as starting points for future analyses, and are hence commonly requested from the authors. However, this mode of data exchange typically requires a time delay for locating, organizing, packaging, and sending data, and can be complicated further by confusion over the way data are named, the type of information they represent, and data formats in which they are stored. Moreover, use of these data in published articles would benefit from the ability to reference a specific and permanent online location, as well as provenance tracking, particularly for purposes of validation and replication. These considerations present a need for a more standardized, easily accessible means of sharing meta-analytic results, which has motivated the creation of a new online data resource called the Archive of Neuroimaging Meta-Analyses (ANIMA). This database can be accessed at http:// anima.fz-juelich.de.

The concept behind the ANIMA database is simple: to provide the results of published meta-analyses and coactivation-based parcellations to interested parties, in the form of statistical maps or labels, encoded as image files. This approach has a number of important requirements. Firstly, data should be easily citable if reused for further analyses, and thus full information about the source of the results must be provided with them. In addition to metadata about the article itself (reference details, cross-links to PubMed entries, abstract information, etc.), there should be sufficient information provided to identify the data

\section{Box 1}

Common methodologies used in ANIMA studies.

Three common approaches used in ANIMA studies are described below.

Activation likelihood estimation (ALE): This is a meta-analytic approach through which peak activation foci, reported in standard MNI or Talairach coordinates, are used to inform Gaussian probability models of activation. This topical approach addresses the sparse information provided by activation foci, as is used to estimate smooth statistical distributions associated with particular psychological or clinical features.

Multi-kernel density analysis (MKDA): A topical approach which is similar in principle to ALE, MKDA uses a spherical instead of Gaussian kernel, and statistical analysis is performed on the number (density) of activation peaks within a given radius.

Meta-analytic connectivity modelling (MACM): This approach models the probability that brain regions are active simultaneously with a particular seed region. MACM is typically performed across many experimental paradigms, providing a meta-analytic estimate of functional connectivity.

Coactivation-based parcellation (CBP): $\mathrm{CBP}$ is a parcellation method derived from co-activation profiles obtained via the MACM approach. CBP uses clustering approaches such as hierarchical or k-means clustering to identify subregions within a larger ROI that are distinct from each other in terms of their connectivity profiles. represented by each image file, including its associated figure, if ap- 102 plicable. Secondly, data retrieved from the database should be simple 103 to organize, visualize, and use. A user should be able to peruse the 104 web interface, query and select studies of interest, download these 105 to their local machine, and immediately browse and utilize the data 106 they have retrieved. Thirdly, in order for the database to expand 107 and provide a thorough sampling of the literature, it is important to 108 provide a convenient interface through which researchers can sub- 109 mit data from their own studies.

With the ANIMA database, we have provided solutions to each of 111 these requirements. In what follows, we will describe our databasing 112 approach, which includes an intuitive online interface for querying, 113 downloading, and submitting data, as well as a stand-alone, cross- 114 platform desktop tool for easily browsing, visualizing, and performing 115 common computations, such as obtaining a conjunction between im- 116 ages. This new resource will provide researchers with a straightforward 117 means of including meta-analytic results in their studies, both as ROIs 118 for future analyses and as a point of comparison against new results. 119 The initial release of the database will include data from 25 published 120 meta-analytic studies, but is intended to grow in order to incorporate 121 the increasing number of studies being added to the literature.

122

\section{Database overview}

ANIMA is designed to serve a number of functions. Firstly, it is a 124 searchable online repository for the results of published neuroimag- 125 ing meta-analyses. Data are organized as individual studies, along 126 with information essential for describing these studies (authors, 127 title, journal, date, abstract, etc.). Included with each study is a set 128 of "study elements", which refer to individual files storing an impor- 129 tant aspect of the study's findings. These will typically be individual 130 volume files, stored in NIFTI format. However, other types of data 131 can be uploaded, including text files containing useful descriptions, 132 image files, or Volume Viewer session files, which store multiple im- 133 ages, along with information on how they should be visualized (see 134 description below). Each study element is also associated with a set 135 of metadata which describe its contents (title, figure captions, type 136 of information, full-text PDF, etc.).

A second function of ANIMA is the ability to submit one's own 138 study to the database. The online interface includes a "Submit" 139 page, which allows registered users to define their study and upload 140 files. If a researcher would like to share results from a meta-analytic 141 study that has been published in peer-reviewed journal, we encour- 142 age them to do so via this interface. The general philosophy of this 143 approach is the concept of "open data". For reasons of security and 144 data integrity, submitting a study requires that a user register with 145 the database, and that each submitted study is first vetted for com- 146 pleteness and validity by the database administrators. However, 147 the process of submitting one's data to ANIMA is designed to be a 148 straightforward process.

Finally, ANIMA was designed with the idea that, once retrieved, 150 data should be simple to organize and query on one's desktop. Ac- 151 cordingly, we have developed a desktop application called Volume 152 Viewer, which interacts seamlessly with the ANIMA interface. Vol- 153 ume Viewer is open source Java-based software tool, built on the 154 ModelGUI API (http://mgui.wikidot.com), and both the program 155 and its source code are freely available online (https://launchpad. 156 net/volumeviewer). The interface allows data to be organized within 157 a "library" framework, in which individual studies can be represent- 158 ed. The data retrieved from ANIMA is already organized according to 159 this framework, so downloaded ANIMA studies can be immediately 160 imported and viewed in Volume Viewer. Furthermore, if a study in- 161 cludes Volume Viewer session files, predefined sets of image com- 162 posites, with custom colour mapping and template or atlas layers, 163 can be viewed with little effort on the part of the user. Importantly, 164 this tool also implements a number of utilities which allow users to 165 
combine their data with ANIMA data, create new ROIs for analyses, report peak coordinates, or quantify the overlap between different distributions.

\section{What data are available?}

At the time of writing, ANIMA contains data from 25 meta-analytic studies (both topic-based ALE meta-analyses and coactivation-based parcellations), as summarized in Table 1 . We intend to expand this number considerably, both by requesting and adding new studies from authors, as well as by encouraging authors to submit their own studies through the online interface. The majority of the data available represent statistical maps which are produced through common meta-analytic approaches, including ALE, CBP, and MACM. Box 1 provides an overview of these methods. In many cases, these data are directly related to a figure in the article, but we also encourage authors to submit supplemental data, if desired. Since the database is based on meta-analyses, no demographic or phenotypical data are directly available, although these can generally be determined by reference to the individual articles. However, the database does provide searchable keywords and sample sizes (number of primary studies, experiments, and subjects) associated with individual articles. Finally, studies in ANIMA each have a unique uniform resource locator (URL), which displays the available data and metadata, along with the date the study was last modified. This URL, along with the modification date, can be used for precise citation of any data used in a new publication. ANIMA keeps a history of any changes to the data that has been made publicly available, such that the exact archive used in a study can be retrieved for purposes of validation or replication.
Data in ANIMA are freely available, although their use is conditional 193 on acceptance of a Data Usage Agreement (DUA). The general terms of 194 this agreement are provided in Box 2. Any redistribution of ANIMA 195 data requires consent from the authors whose data are redistributed, 196 as well as the database owners. Additionally, the DUA specifies that at- 197 tribution for use of the data must include reference to both the database 198 and the specific studies used. Both database users and contributors must 199 agree to the terms of the DUA in order to use or contribute to the data- 200 base, respectively.

\section{Accessing, viewing, and using ANIMA data}

Fig. 1 shows the online interface for browsing studies in ANIMA 203 (http://anima.fz-juelich.de/index.php). Notably, querying and 204 downloading is a fully open process with no registration required. Stud- 205 ies are listed in tabular format, with each entry showing the title of the 206 study (in the form of the lead author and publication year), a brief de- 207 scription, the size of the archive, and links to download the study, 208 view its PubMed page, or download its associated full-text PDF. At the 209 top of the page, a search bar allows the user to search by author, publi- 210 cation year, or journal. More detailed information about individual 211 studies can be accessed by clicking on the study's title. Here, abstract, 212 keywords, and version information are displayed, as well as a list of in- 213 dividual study elements (Fig. 2). It is also possible to view image ele- 214 ments, using the Javascript-based Papaya viewer (http://github.com/ 215 rii-mango/Papaya), by clicking on the eye icon for an individual ele- 216 ment. This will overlay the image on the non-linear ICBM-152 anatom- 217 ical template, and is accessible from any modern web browser.

Table 1

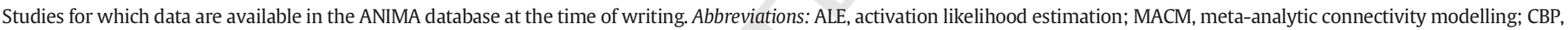
coactivation-based parcellation; RS-fMRI, resting-state fMRI connectivity.

\begin{tabular}{|c|c|c|}
\hline Study & Description & Methods \\
\hline Amft et al. (2014) & Characterized an extended social-affective default network through coactivation-based parcellation & MACM, RS-fMRI, CBP \\
\hline Bzdok et al. (2011) & $\begin{array}{l}\text { Performed meta-analysis to investigate the localization of facial judgments of trustworthiness and } \\
\text { attractiveness }\end{array}$ & ALE \\
\hline Bzdok et al. (2012b) & Performed ALE meta-analysis on morality, theory of mind, and empathy & ALE \\
\hline Bzdok et al. (2013a) & Investigated the structural, connectional, and functional subspecialization of the amygdala & MACM, CBP \\
\hline Bzdok et al. (2013b) & Investigated parcellation, connectivity, and functional decoding of the temporoparietal junction & MACM, RS-fMRI, CBP \\
\hline Caspers et al. (2010) & $\begin{array}{l}\text { Performed meta-analysis to investigate the localization of action observation and imitation in the } \\
\text { human brain }\end{array}$ & ALE \\
\hline Cieslik et al. (2013) & Derived a coactivation-based parcellation of the dorsolateral prefrontal cortex & MACM, RS-fMRI, CBP \\
\hline Cieslik et al. (2015) & Identified three key regions for supervisory attentional control, using meta-analysis & ALE \\
\hline Clos et al. (2013) & $\begin{array}{l}\text { Used coactivation-based parcellation to divide Broca's area into five clusters, each having distinct } \\
\text { functional profiles }\end{array}$ & MACM, CBP \\
\hline Friebel et al. (2011) & Performed an ALE meta-analysis of experimentally induced and chronic persistent neuropathic pain & ALE \\
\hline Goodkind et al. (in press) & $\begin{array}{l}\text { Combined meta-analysis of VBM studies across psychiatric disorders, with three parallel } \\
\text { connectivity analyses }\end{array}$ & ALE, MACM \\
\hline Hardwick et al. (2013) & Performed meta-analysis to investigate the localization of motor learning in the human brain & ALE \\
\hline Hoffstaedter et al. (2014) & $\begin{array}{l}\text { Compared functional connectivity derived from RS-fMRI and MACM, for the anterior mid-cingulate } \\
\text { cortex }\end{array}$ & MACM, RS-fMRI \\
\hline Keuken et al. (2014) & Used meta-analysis to characterize the brain regions associated with perceptual decision making & ALE \\
\hline Kohn et al. (2014) & Performed an ALE meta-analysis and MACM analysis of cognitive emotion regulation & ALE, MACM \\
\hline Krall et al. (2014) & $\begin{array}{l}\text { Performed an ALE meta-analysis to investigate the role of the right temporoparietal junction in both } \\
\text { attention and social interaction }\end{array}$ & ALE \\
\hline Kurth et al. (2010) & $\begin{array}{l}\text { Performed an ALE meta-analysis to investigate the functional differentiation and integration of the } \\
\text { human insula }\end{array}$ & ALE \\
\hline Langner and Eickhoff (2013) & Conducted a meta-analytic review of the neural mechanisms of vigilant (sustained) attention & ALE \\
\hline Müller et al. (2014) & $\begin{array}{l}\text { Investigated the influence of gray matter volume, functional connectivity and trait impulsivity on } \\
\text { cognitive flexibility }\end{array}$ & ALE \\
\hline Nellessen et al. (2014) & $\begin{array}{l}\text { Performed an ALE meta-analysis on } 28 \text { task-based fMRI studies, in patients with mild cognitive } \\
\text { impairment or Alzheimer's disease }\end{array}$ & ALE \\
\hline Nickl-Jockschat et al. (2014) & $\begin{array}{l}\text { Investigated the neural correlates of face processing in subjects with autism spectrum disorder } \\
\text { (ASD), using ALE meta-analysis }\end{array}$ & ALE \\
\hline Reid et al. (2015) & $\begin{array}{l}\text { Compared MACM, RS-fMRI, and structural covariance to investigate the convergence of connectivity } \\
\text { estimates for the anterior lateral prefrontal cortex (aLPFC) }\end{array}$ & MACM, RS-fMRI \\
\hline Rottschy et al. (2012) & Investigated the neural correlates of working memory using ALE meta-analysis & ALE \\
\hline Schilbach et al. (2012) & $\begin{array}{l}\text { Used ALE meta-analyses to study the neural correlates of emotional processing, social \& default } \\
\text { mode cognition }\end{array}$ & ALE \\
\hline zu Eulenburg et al. (2012) & $\begin{array}{l}\text { Used meta-analysis to localize activations corresponding to a vestibular stimulus, and evaluated } \\
\text { functional connectivity of these regions using RS-fMRI }\end{array}$ & ALE, RS-fMRI \\
\hline
\end{tabular}




\section{Box 2}

Summary of the data usage agreement for the ANIMA database.

- Data are provided "as is", with no guarantee whatsoever

- Data can be used for any non-commercial purpose, with the exception that redistribution of the data can only be done if written consent is obtained from the database owners, and only for studies whose authors have approved redistribution.

- Any public use of ANIMA data must provide attribution, specifically by reference to the database itself, and the primary article associated with those data

- The owners of ANIMA do not claim any copyright over the data archived in the database

The web page allows a user to download single studies, or select and download multiple selected studies at once. The download will be a compressed tar archive, which can be decompressed and either used directly in one's analyses, or viewed and queried using the Volume Viewer tool (Fig. 3). This is facilitated through the inclusion of XML-format files which store study metadata and a list of study elements, along with the actual data. Image files are stored in NIFTI format (although Volume Viewer can also read MINC or MGZ format images). Volume Viewer provides a convenient dialog for automatically interpolating images which have been registered to the same space, but which do not have the same size or orientation as the current volume. This allows comparison of multiple images without having to preprocess them in advance. The process of downloading and visualizing a study is illustrated in Fig. 4.

Volume Viewer currently supports a number of useful features which provide supplemental utility to the ANIMA online interface. These include:

- The ability to overlay and compare images. This allows a user, for instance, to compare the results of a new analysis with those of published meta-analyses in ANIMA.
- The ability to save the current configuration as a single Volume View- 238 er session file (".vvs" extension), which can be uploaded to ANIMA via 239 the online interface, and viewed by all users who download a given 240 study. The session includes all images, templates, and atlases, the 241 way they are arranged and composited, and the currently selected 242 viewing planes.

- The ability to compute a conjunction image, and quantify the degree 244 of overlap between two images. This feature also yields ROIs that 245 can be immediately used for further analysis.

- The ability to obtain peak coordinates from smoothed maps, for 247 reporting in an article, or use as seed points in further analyses. $\quad 248$

- The inclusion of standard template and atlas images, which allow data 249 to be cross-referenced against anatomy or well-known parcellation 250 schemes. A dialogue for defining new atlases is also provided.

\section{Submitting your study to ANIMA}

ANIMA provides an intuitive online interface for submitting a study 254 for inclusion in the database (http://anima.fz-juelich.de/submit.php). 255 The submission process follows a series of tabs, as shown in Fig. 5. The 256 first tab ("Login") allows a new user to register with the database, 257 which is necessary to submit data. Registration allows the administra- 258 tors of ANIMA to associate a name and institution with a particular 259 study, and provides a level of security by ensuring users provide a 260 valid email address. The email address will not be accessible to the pub- 261 lic, and will only be used to contact the user in case of issues related to 262 the study. Registered users can also log in using this tab. The next step 263 of the process (the "Study" tab) requires the user to enter meta-data 264 about the study. This includes author and publication information, a 265 brief description (for browsing purposes), and a full-text PDF, if desired 266 and permitted. The subsequent tab ("Elements") provides a means of 267 uploading the study elements (i.e., data files), and associated titles and 268 captions. Four types of files can be uploaded, as shown in Table 2. The 269 final tab ("Submit") provides a preview of the study as entered, and 270

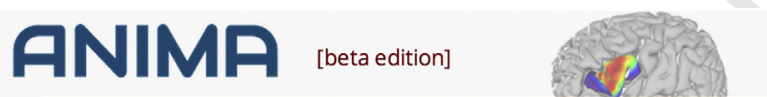

WELCOME

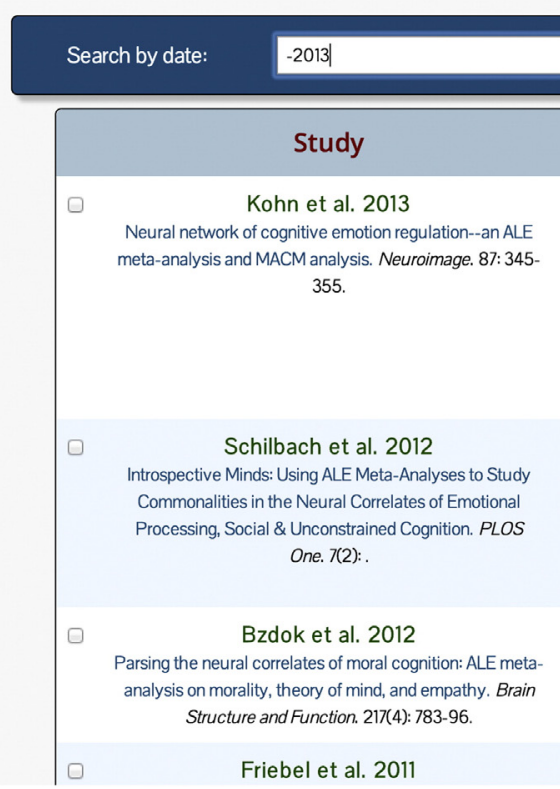

We here quantitatively summarize the published literature on cognitive emotion regulation using activation likelihood estimation in $\mathrm{FMRI}$ and PET ( 23 studies/479 subjects). According to our model, the superior temporal gyrus, angular gyrus and (pre) supplementary motor area should be involved in execution of regulation initiated by frontal areas. The dorsolateral prefrontal cortex may be related to regulation of cognitive processes such as attention, while the ventrolateral prefrontal cortex may not necessarily reflect the regulatory process per se, but signals salience and therefore the need to regulate.

We present results of quantitative meta-analyses of neuroimaging studies, which confirm a statistical convergence in the neural correlates of social and resting state cognition. By using conjunction analyses across studies investigating social cognition, resting state, and emotion, we demonstrate significant overlap of task-related signal change in dorso-medial prefrontal and medial parietal cortex, brain regions that have recently been linked to introspective abilities.

We tested the hypothesis that moral decisions might be implemented in brain areas engaged in "theory of mind" and empathy, by conducting a large-scale activation likelihood estimation (ALE) meta-analysis of neuroimaging studies, which assessed 2,607 peak coordinates from 247 experiments in 1,790 participants.

We performed a meta-analysis based on a literature search of published functional
(1)

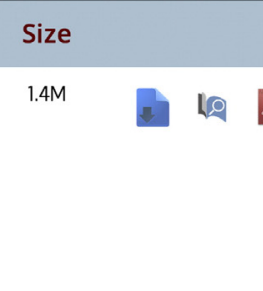

Lo

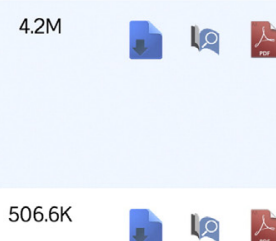

L

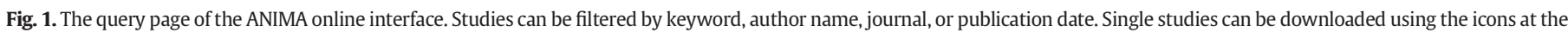
right of each entry, and multiple studies can also be selected and downloaded together using the check boxes at left. 


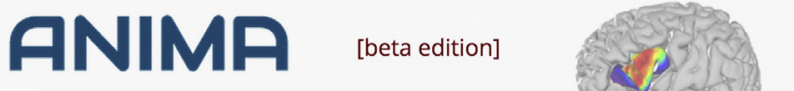

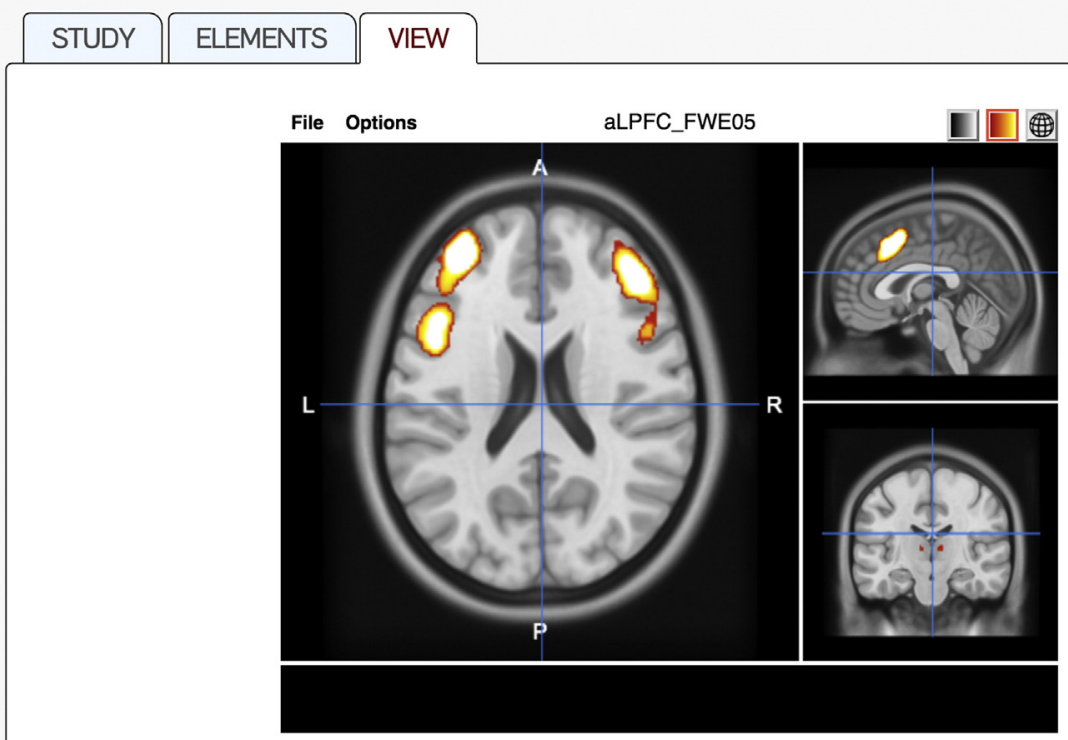

Figure 2A

MACM result for aLPFC

Quantity: T-statistic

Coordinate space: ICBM-152

Threshold method: FWE corrected

Fig. 2. The single-study information page of the ANIMA online interface, showing the Papaya viewer. This page displays more detailed information about a study, including a list of individual study elements and their associated captions.

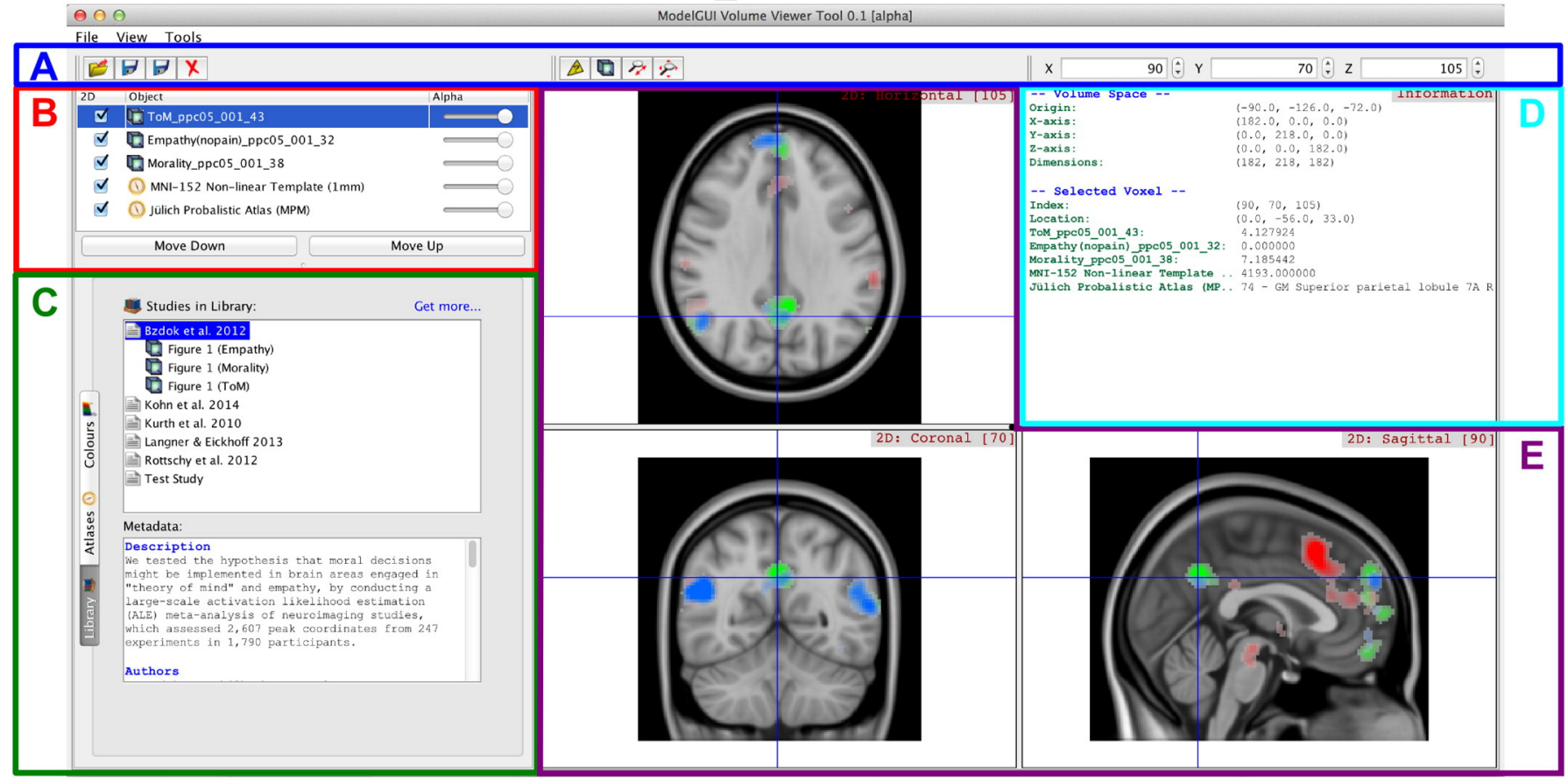

Fig. 3. Screenshot of the stand-alone desktop application Volume Viewer. A. The toolbar, which allows individual images or Volume Viewer sessions to be loaded or saved, or removed from the current session. Buttons in the middle of the panel allow the user to set the mouse behaviour (zoom or query mode). At far right, the current voxel coordinates are display and can be modified. B. The image selection list, which allows the visibility of individual layers to be toggled, their order to be modified, and their transparency (alpha) to be set. C. The Library Panel, which lists the user's local library of studies which have already been downloaded. Individual study elements (images or entire Volume Viewer sessions) can be loaded via this panel. Two other panels are also selectable in this space: (i) the Atlases panel, which allows individual standard atlas or template images to be loaded; and (ii) the Colours panel (shown in inset), which allows a predefined colour map to be applied to the currently selected image layer. D. The Information Panel, which displays information about the current volume space, as well as the voxel defined by the currently viewed sections. Values can be queried for numeric data, while text representations are shown for atlases (here the Maximum Probability Map of the Jülich Probabilistic Atlas). E. The 2D rendering panels, which show the three standard orthogonal planes of the loaded images (horizontal/transversal, coronal, and sagittal). These can be zoomed, panned, and queried with the mouse. 


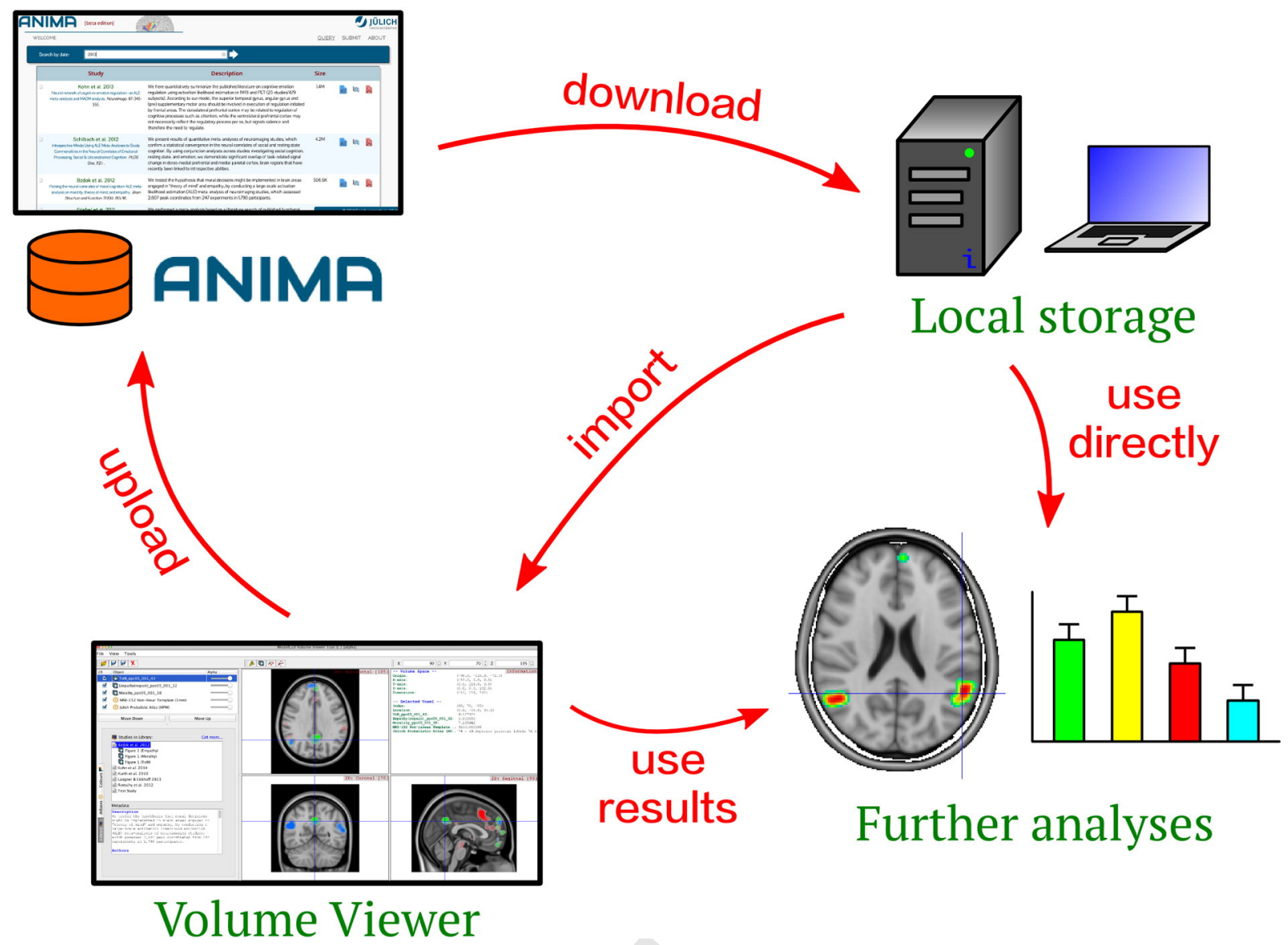

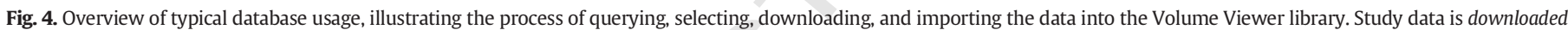

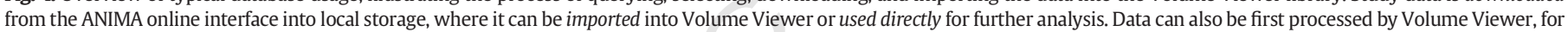
instance to extract conjunction ROIs, before being used in further analyses. Volume Viewer can also be used to defined sessions, which can be uploaded to the database.

allows the user to verify all information before submitting it to the database.

Upon submission, a message will be sent to the database curators, who will review the submission for validity, completeness, typographic errors, and any other issues. If issues are encountered, an email will be sent with instructions to the submitter on how to update them. Otherwise, the study will be accepted and a confirmation email will be sent. This curation design ensures that only valid, published data are made available through ANIMA, and that the uploader has the right to share data on behalf of the associated authors. Notably, once a study is submitted, it is always associated with the submitter. Modifications can then be made to the study only by the submitter or a database administrator. ANIMA provides a "subscribe" feature, which allows the submitter (as well as any registered user) to request a notification whenever a specific study is modified. Any modifications made to the study (including edits, additions, subtractions, or removal) will result in subscribers being automatically notified of the change via email. Additionally, ANIMA implements a simple versioning policy, which ensures that any major modifications to existing studies are captured as new versions of the study. This feature is important, since any publication using ANIMA data should be able to point to the precise version of a data element used for its analysis.

\section{Metadata}

The use of metadata to precisely define individual neuroimaging results is an important consideration for any online database, and is essential for both understanding individual database elements, and facilitating further meta-analyses (Poldrack and Gorgolewski, 2014). For the ANIMA database, all such metadata are encoded in XML format, 298 which allows them to be easily extended as necessary. In its present 299 state, the database provides metadata for whole studies (including cita- 300 tion details, keywords, abstracts, and DOIs), as well as single study ele- 301 ments. Study element metadata include a caption which describes the 302 element, and - specifically for image data - further details such as the 303 quantity represented, how the image is thresholded, and the coordinate 304 space (standard or native) in which it is expressed.

\section{Future plans}

The platform described above was designed to provide simple, effi- 307 cient access to results of a specific type of study (meta-analyses), in a 308 standard way. Moreover, the inclusion of a stand-alone desktop applica- 309 tion provides a useful means of visualizing, querying, and manipulating 310 data for use in future studies. However, both the online and standalone 311 components of this platform are also generic enough to be used with 312 other types of studies as well. All data is stored as XML files, which 313 can be easily extended or modified, depending on the type of data re- 314 quired. One potential future development will be to host other types 315 of study results using the ANIMA interface. Ideally, this will involve ex- 316 posing the database code as a documented and supported open-source 317 project, and providing an online REST interface accessible to registered 318 users (Masse, 2011). As an extension of this, ways in which ANIMA 319 could be integrated with existing databases will also be pursued. Specif- 320 ically, we plan to implement a REST API in order to allow a database 321 such as Neurovault.org (Gorgolewski et al., 2015) to access ANIMA 322 data, for inclusion in large-scale meta-analytic approaches. 
A

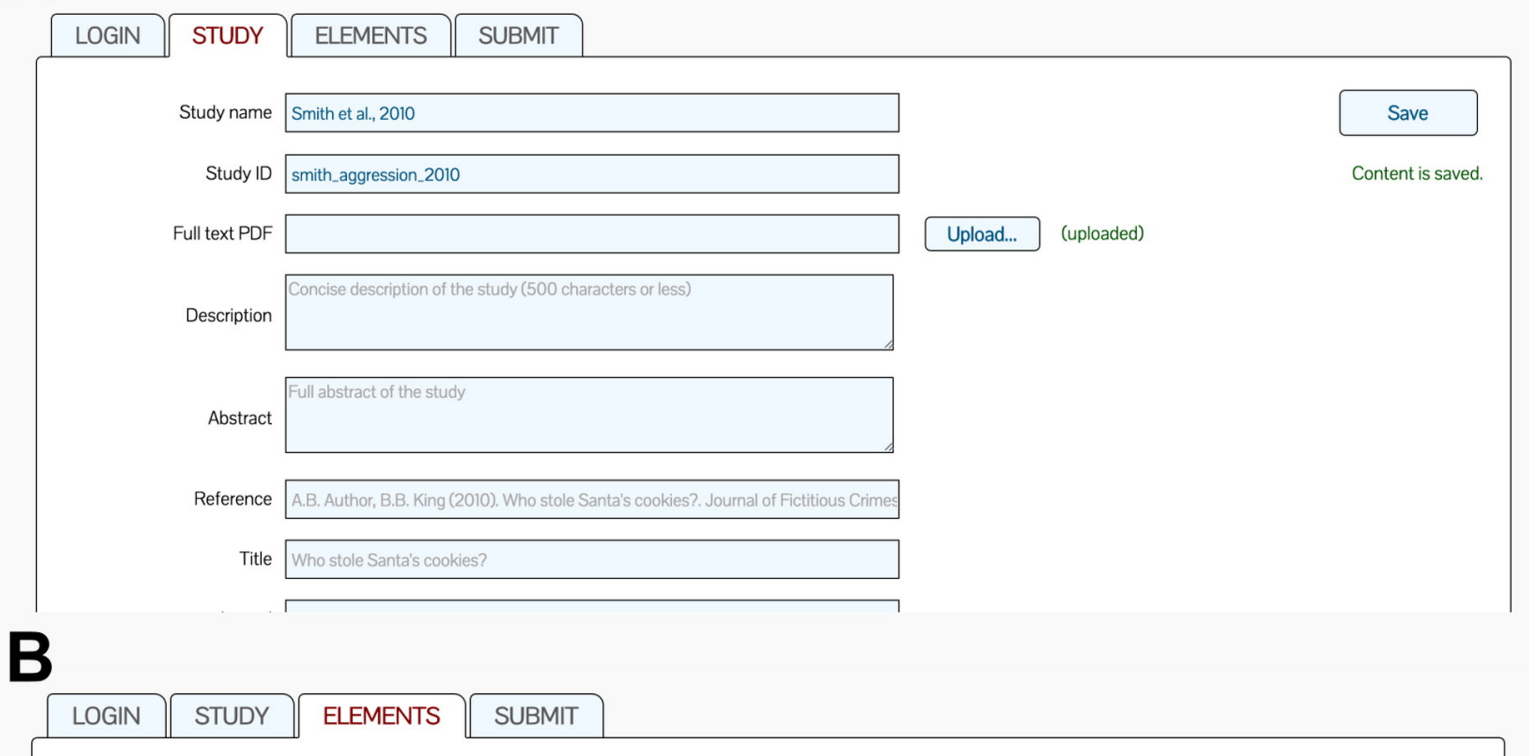

Enter a new study element

Name Figure 2

Type Volume file (nii, mnc, mgz)

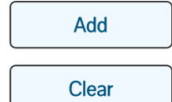

Caption 4

File NbackSternbergVerbalNonverbal_andMore_3Phasen_thr20.nii.gz $\quad$ Upload...

Coordinates ICBM-152 *

Quantity T-statistic

Threshold None $\quad$ ज

\begin{tabular}{|llll}
\hline Name & Type & Caption & Actions
\end{tabular}

C

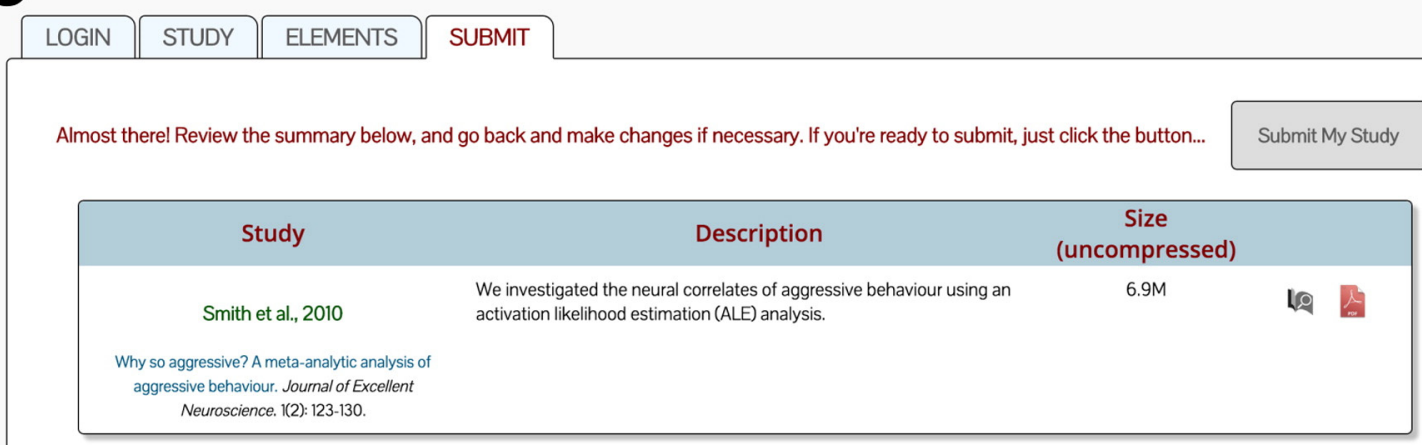

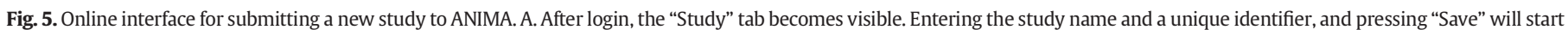

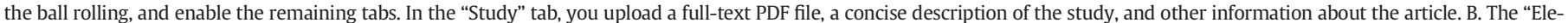

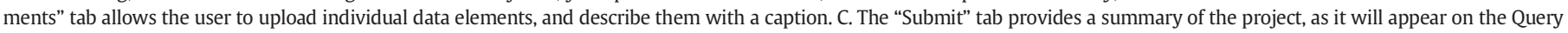

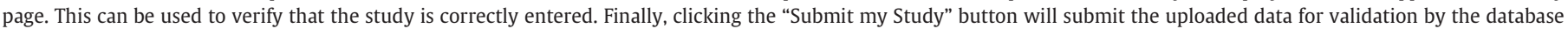
administrators.

Another important consideration is how the database will be maintained and managed in the future. The organization of the database ensures that its management is minimal, but it is still important to have a team of administrators who can evaluate submissions, respond to feed- 327 back about data quality, and provide support to users of the database. 328 We intend to assume this role for the foreseeable future. As an extension 329 
t4.1 Table 2

t4.2 File types which can be uploaded to ANIMA.

$\mathrm{t} 4.3$

$\mathrm{t} 4.4$

File type
Volume File
Volume Viewer Session File

Volume Viewer Point Set File

Image File

Text File
Description

A 3D brain image file, typically containing statistical maps such as t-, z-, or p-scores, which indicate the result of a specific analysis. These should be in NIFTI format. An XML-format file containing all data and metadata specifying a Volume Viewer session. This can be used to pre-define a set of composite images, templates, atlases, and their colour mapping. An XML-format file specifying a list of 3D coordinates, along with associated data which can be used to specify and label individual points of interest in a study. A standard 2D image file, which could represent an informative figure, schematic, or other useful information pertaining to the study.

A text file, which can contain additional free descriptions or other information about the study and its elements.
Extensions

nii, hdr, nii.gz

Vvs

pointset

png, jpg, gif, tif, etc.

txt, rtf of this, additional support for ANIMA will be provided in terms of documentation, and an online support forum which can be used to ensure that common issues can be answered once, and a means of reporting bugs, which can be of great assistance to the database developers.

The Volume Viewer tool is based on the ModelGUI API, which is an open-source project hosted on http://www.launchpad.net/volumeviewer (an online platform designed to be scalable, and which supports projects as large as the Ubuntu community). We intend to provide Volume Viewer also as an open-source project, such that it can be freely obtained and developed by interested members of the neuroscience community. A number of future improvements are planned for Volume Viewer, including: the ability to specify named voxels and ROIs; the ability to extract peaks from a smoothed image map; the addition of 3D volume and surface rendering; easy transfer of data between surfaces and volumes; rendering of network graphs for the visualization of connectivity information; and layout and printing features.

\section{Summary}

We present a new database which provides free and convenient online access to the results of published neuroimaging meta-analyses. Data can be used for comparison with one's own results, or as a starting point for new analyses, and the ANIMA interface provides a set of simple tools which greatly facilitates this process. This interface includes a search function, a form for submitting new studies, and an opensource stand-alone software tool for visualizing and organizing study data, and generating ROIs for further analysis. It is our hope that the ANIMA database will improve the way in which the results of metaanalytic neuroimaging studies will be used in the future, and encourage researchers to incorporate these important results into their research approaches.

\section{References}

Amft, M., Bzdok, D., Laird, A.R., Fox, P.T., Schilbach, L., Eickhoff, S.B., 2014. Definition and characterization of an extended social-affective default network. Brain Struct. Funct. http://dx.doi.org/10.1007/s00429-013-0698-0.

Bzdok, D., Laird, A.R., Zilles, K., Fox, P.T., Eickhoff, S.B., 2013a. An investigation of the structural, connectional, and functional subspecialization in the human amygdala. Hum. Brain Mapp. 34, 3247-3266. http://dx.doi.org/10.1002/hbm.22138.

Bzdok, D., Langner, R., Caspers, S., Kurth, F., Habel, U., Zilles, K., Laird, A., Eickhoff, S.B. 2011. ALE meta-analysis on facial judgments of trustworthiness and attractiveness. Brain Struct. Funct. 215, 209-223. http://dx.doi.org/10.1007/s00429-0100287-4.

Bzdok, D., Langner, R., Schilbach, L., Jakobs, O., Roski, C., Caspers, S., Laird, A.R., Fox, P.T., Zilles, K., Eickhoff, S.B., 2013b. Characterization of the temporo-parietal junction by combining data-driven parcellation, complementary connectivity analyses, and functional decoding. Neuroimage 81, 381-392. http://dx.doi.org/10.1016/j.neuroimage. 2013.05.046

Bzdok, D., Schilbach, L., Vogeley, K., Schneider, K., Laird, A.R., Langner, R., Eickhoff, S.B., 2012. Parsing the neural correlates of moral cognition: ALE meta-analysis on morality, theory of mind, and empathy. Brain Struct. Funct. 217, 783-796.

Caspers, S., Zilles, K., Laird, A.R., Eickhoff, S.B., 2010. ALE meta-analysis of action observation and imitation in the human brain. Neuroimage 50, 1148-1167. http://dx.doi.org/ 10.1016/j.neuroimage.2009.12.112.
Chang, L.J., Yarkoni, T., Khaw, M.W., Sanfey, A.G., 2012. Decoding the role of the insula in 381 human cognition: functional parcellation and large-scale reverse inference. Cereb. 382 Cortex http://dx.doi.org/10.1093/cercor/bhs065 (bhs065). 383

Cieslik, E.C., Mueller, V.I., Eickhoff, C.R., Langner, R., Eickhoff, S.B., 2015. Three key regions for 384 supervisory attentional control: evidence from neuroimaging meta-analyses. Neurosci. 385 Biobehav. Rev. 48C, 22-34. http://dx.doi.org/10.1016/j.neubiorev.2014.11.003. 386

Cieslik, E.C., Zilles, K., Caspers, S., Roski, C., Kellermann, T.S., Jakobs, O., Langner, R., Laird, 387 A.R., Fox, P.T., Eickhoff, S.B., 2013. Is there "one" DLPFC in cognitive action control? Ev- 388 idence for heterogeneity from co-activation-based parcellation. Cereb. Cortex 23, 389 2677-2689. http://dx.doi.org/10.1093/cercor/bhs256.

Clos, M. Amunts, K. Laird, A.R, Fox, PT . Eickhoff, S.B. 2013. Tackling the multifunctional 391 nature of Broca's region meta-analytically: co-activation-based parcellation of area 392 44. Neuroimage 83, 174-188. http://dx.doi.org/10.1016/j.neuroimage.2013.06.041. 393

Eickhoff, S.B., Bzdok, D., Laird, A.R., Kurth, F., Fox, P.T., 2012. Activation likelihood estima- 394 tion meta-analysis revisited. Neuroimage 59, 2349-2361. http://dx.doi.org/10.1016/j. 395 neuroimage.2011.09.017.

Eickhoff, S.B., Bzdok, D., Laird, A.R., Roski, C., Caspers, S., Zilles, K., Fox, P.T., 2011. Co- 397 activation patterns distinguish cortical modules, their connectivity and functional 398 differentiation. Neuroimage 57, 938-949. http://dx.doi.org/10.1016/j.neuroimage. 399 2011.05.021.

Eickhoff, S.B., Laird, A.R., Grefkes, C., Wang, L.E.,Zilles, K., Fox, P.T., 2009. Coordinate-based 401 activation likelihood estimation meta-analysis of neuroimaging data: a random- 402 effects approach based on empirical estimates of spatial uncertainty. Hum. Brain 403 Mapp. 30, 2907-2926. http://dx.doi.org/10.1002/hbm.20718. 404

Etkin, A., Wager, T.D., 2007. Functional neuroimaging of anxiety: a meta-analysis of emo- 405 tional processing in PTSD, social anxiety disorder, and specific phobia. AJP 164, 406 1476-1488. http://dx.doi.org/10.1176/appi.ajp.2007.07030504. 407

Fox, P.T., Lancaster, J.L., Laird, A.R., Eickhoff, S.B., 2014. Meta-analysis in human neuroim- 408 aging: computational modeling of large-scale databases. Annu. Rev. Neurosci. 37, 409 409-434. http://dx.doi.org/10.1146/annurev-neuro-062012-170320. 410

Friebel, U., Eickhoff, S.B., Lotze, M., 2011. Coordinate-based meta-analysis of experimen- 411 tally induced and chronic persistent neuropathic pain. Neuroimage 58, 1070-1080. 412 http://dx.doi.org/10.1016/j.neuroimage.2011.07.022.

Goodkind, M., Eickhoff, S.B., Oathes, D., Jiang, Y., Chang, A., Jones-Hagata, L., Ortega, B.N., 414 Zaiko, Y.V., Roach, E.L., Korgaonkar, M.S., Grieve, S.M., Galatzer-Levy, I., Fox, P.T., 415 Etkin, A., 2015. Identification of a common neurobiological substrate for mental ill- 416 ness. JAMA Psychiatry (in press).

Gorgolewski, K.J., Varoquaux, G., Rivera, G., Schwarz, Y., Ghosh, S.S., Maumet, C., Sochat, 418 V.V., Nichols, T.E., Poldrack, R.A., Poline, J.-B., Yarkoni, T., Margulies, D.S., 2015. 419 NeuroVault.org: a web-based repository for collecting and sharing unthresholded 420 statistical maps of the human brain. Front. Neuroinform. 9, 8. http://dx.doi.org/10. 421 3389/fninf.2015.00008.

Hardwick, R.M., Rottschy, C., Miall, R.C., Eickhoff, S.B., 2013. A quantitative meta-analysis 423 and review of motor learning in the human brain. Neuroimage 67, 283-297. http:// 424 dx.doi.org/10.1016/j.neuroimage.2012.11.020. 425

Hoffstaedter, F., Grefkes, C., Caspers, S., Roski, C., Palomero-Gallagher, N., Laird, A.R., Fox, 426 P.T., Eickhoff, S.B., 2014. The role of anterior midcingulate cortex in cognitive motor 427 control: evidence from functional connectivity analyses. Hum. Brain Mapp. 35, 428 2741-2753. http://dx.doi.org/10.1002/hbm.22363.

Keuken, M.C., Müller-Axt, C., Langner, R., Eickhoff, S.B., Forstmann, B.U., Neumann, J., 430 2014. Brain networks of perceptual decision-making: an fMRI ALE meta-analysis. 431 Front. Hum. Neurosci. 8. http://dx.doi.org/10.3389/fnhum.2014.00445.

Kober, H., Barrett, L.F., Joseph, J., Bliss-Moreau, E., Lindquist, K., Wager, T.D., 2008. 433 Functional grouping and cortical-subcortical interactions in emotion: a meta- 434 analysis of neuroimaging studies. Neuroimage 42, 998-1031. http://dx.doi.org/ 435 10.1016/j.neuroimage.2008.03.059.

Kohn, N., Eickhoff, S.B., Scheller, M., Laird, A.R., Fox, P.T., Habel, U., 2014. Neural network 437 of cognitive emotion regulation-an ALE meta-analysis and MACM analysis. 438 Neuroimage 87, 345-355. http://dx.doi.org/10.1016/j.neuroimage.2013.11.001. 439

Kurth, F., Zilles, K., Fox, P.T., Laird, A.R., Eickhoff, S.B., 2010. A link between the systems: func- 440 tional differentiation and integration within the human insula revealed by meta- 441 analysis. Brain Struct. Funct. 214, 519-534. http://dx.doi.org/10.1007/s00429-010- 442 0255-z.

Laird, A.R., Eickhoff, S.B., Fox, P.M., Uecker, A.M., Ray, K.L., Saenz, J.J., McKay, D.R., Bzdok, 444 D., Laird, R.W., Robinson, J.L., Turner, J.A., Turkeltaub, P.E., Lancaster, J.L., Fox, P.T., 445 2011. The BrainMap strategy for standardization, sharing, and meta-analysis of neu- 446 roimaging data. BMC Res. Notes 4, 349. http://dx.doi.org/10.1186/1756-0500-4-349. 447 
Langner, R., Eickhoff, S.B., 2013. Sustaining attention to simple tasks: a meta-analytic review of the neural mechanisms of vigilant attention. Psychol. Bull. 139, 870-900. http://dx.doi.org/10.1037/a0030694

Masse, M., 2011. REST API Design Rulebook. O'Reilly Media, Inc.

Müller, V.I., Langner, R., Cieslik, E.C., Rottschy, C., Eickhoff, S.B., 2014. Interindividual differences in cognitive flexibility: influence of gray matter volume, functional connectivity and trait impulsivity. Brain Struct. Funct. http://dx.doi.org/10. 1007/s00429-014-0797-6.

Nellessen, N., Rottschy, C., Eickhoff, S.B., Ketteler, S.T., Kuhn, H., Shah, N.J., Schulz, J.B., Reske, M., Reetz, K., 2014. Specific and disease stage-dependent episodic memoryrelated brain activation patterns in Alzheimer's disease: a coordinate-based metaanalysis. Brain Struct. Funct. http://dx.doi.org/10.1007/s00429-014-0744-6.

Nickl-Jockschat, T., Rottschy, C., Thommes, J., Schneider, F., Laird, A.R., Fox, P.T., Eickhoff, S.B. 2014. Neural networks related to dysfunctional face processing in autism spectrum disorder. Brain Struct. Funct. 1-17. http://dx.doi.org/10.1007/s00429-014-0791-z.

Northoff, G., Heinzel, A., de Greck, M., Bermpohl, F., Dobrowolny, H., Panksepp, J., 2006. Self-referential processing in our brain-a meta-analysis of imaging studies on the self. Neurolmage 31, 440-457. http://dx.doi.org/10.1016/j.neuroimage.2005.12.002.

Poldrack, R.A., Gorgolewski, K.J., 2014. Making big data open: data sharing in neuroimaging. Nat. Neurosci. 17, 1510-1517. http://dx.doi.org/10.1038/nn.3818.

Reid, A.T., Bzdok, D., Langner, R., Fox, P.T., Laird, A.R., Amunts, K., Eickhoff, S.B., Eickhoff, C.R., 2015. Multimodal connectivity mapping of the human left anterior and posterior lateral prefrontal cortex. Brain Struct. Funct. 1-17. http://dx.doi.org/10.1007/s00429015-1060-5.

Robinson, J.L., Laird, A.R., Glahn, D.C., Lovallo, W.R., Fox, P.T., 2010. Metaanalytic connectivity modeling: delineating the functional connectivity of the human amygdala. Hum. Brain Mapp. 31, 173-184. http://dx.doi.org/10.1002/hbm.20854.
Rottschy, C., Langner, R., Dogan, I., Reetz, K., Laird, A.R., Schulz, J.B., Fox, P. T. 475 Eickhoff, S.B., 2012. Modelling neural correlates of working memory: a 476 coordinate-based meta-analysis. Neuroimage 60, 830-846. http://dx.doi.org/ 477 10.1016/j.neuroimage.2011.11.050.

Schilbach, L., Bzdok, D., Timmermans, B., Fox, P.T., Laird, A.R., Vogeley, K., Eickhoff, S.B., 479 2012. Introspective minds: using ALE meta-analyses to study commonalities in the 480 neural correlates of emotional processing, social \& unconstrained cognition. PLoS 481 ONE 7, e30920. http://dx.doi.org/10.1371/journal.pone.0030920.

Schilbach, L., Müller, V.I., Hoffstaedter, F., Clos, M., Goya-Maldonado, R., Gruber, O., 483 Eickhoff, S.B., 2014. Meta-analytically informed network analysis of resting state 484 FMRI reveals hyperconnectivity in an introspective socio-affective network in depres- 485 sion. PLoS ONE 9, e94973. http://dx.doi.org/10.1371/journal.pone.0094973. 486

Wager, T.D., Lindquist, M., Kaplan, L., 2007. Meta-analysis of functional neuroimaging 487 data: current and future directions. Soc. Cogn. Affect. Neurosci. 2, 150-158. http:// 488 dx.doi.org/10.1093/scan/nsm015.

Xue, W., Kang, J., Bowman, F.D., Wager, T.D., Guo, J., 2014. Identifying functional co- 490 activation patterns in neuroimaging studies via poisson graphical models. Biom 70, 491 812-822. http://dx.doi.org/10.1111/biom.12216.

Yarkoni, T., Poldrack, R.A., Nichols, T.E., Van Essen, D.C., Wager, T.D., 2011. Large-scale au- 493 tomated synthesis of human functional neuroimaging data. Nat. Methods 8, 665-670. 494 http://dx.doi.org/10.1038/nmeth.1635.

Eulenburg Zu, Caspers, P., Roski, S., Eickhoff, C., S.B., 2012. Meta-analytical definition and 496 functional connectivity of the human vestibular cortex. Neuroimage 60, 162-169. 497 http://dx.doi.org/10.1016/j.neuroimage.2011.12.032 